\title{
Characterization of an Enzyme-Catalyzed Crosslinkable Hydrogel as a Wound Dressing in Skin Tissue Engineering
}

\author{
Mohammad Ali Nilforoushzadeh ${ }^{1,2}{ }^{\circledR}$, Amir Behtash Amiri', Behrad Shaghaghi ${ }^{3}{ }^{\circledR}$, Alimohammad \\ Alimohammadi $^{\circledR}$, Rahim Ahmadi ${ }^{{ }^{\circledR}}$, Ebrahim Khodaverdi Darian ${ }^{6,7}$, Mohammadreza Razzaghi ${ }^{\circledR}$, \\ Mostafa Rezaei Tavirani ${ }^{\mathbb{D}}$, Sona Zare ${ }^{1,8^{*} \mathbb{D}}$ \\ ${ }^{1}$ Skin and Stem Cell Research Center, Tehran University of Medical Sciences, Tehran, Iran \\ ${ }^{2}$ Jordan Dermatology and Hair Transplantation Center, Tehran, Iran \\ ${ }^{3}$ Polymer Laboratory, School of Chemistry, College of Science, University of Tehran, Tehran, Iran \\ ${ }^{4}$ Forensic Medicine Specialist, Research Center of Legal Medicine Organization of Iran, Tehran, Iran \\ ${ }^{5}$ Department of Biology, Hamedan Branch, Islamic Azad University, Hamedan, Iran \\ ${ }^{6}$ Department of Medical Biotechnology, School of Medicine, Semnan University of Medical Sciences, Semnan, Iran \\ ${ }^{7}$ Biotechnology Research Center, Semnan University of Medical Sciences, Semnan, Iran \\ ${ }^{8}$ Laser Application in Medical Sciences Research Center, Shahid Beheshti University of Medical Sciences, Tehran, Iran \\ ${ }^{9}$ Proteomics Research Center, Faculty of Paramedical Sciences, Shahid Beheshti University of Medical Sciences, \\ Tehran, Iran
}

\section{*Correspondence to Sona Zare, PhD; Skin and Stem Cell Research Center, Tehran University of Medical Sciences, Tehran, Iran. Email: sonazarebio@gmail.com}

Received: December 27, 2020 Accepted: August 23, 2021 Published online December 1, 2021

\begin{abstract}
Introduction: Wound healing can have a very important impact on the patients' quality of life. For its treatment, wound dressings have vital and effective uses. Indeed, the use of a proper wound dressing can improve the healing process and duration. Recently, wound dressings with unique properties have been prepared using natural hydrogels. In addition to the general wound characteristics, new generations of wound dressings, such as those lasting longer on the wound, can have specific properties such as transferring allogeneic cells to enhance the healing effect and speed up the healing process. The present study aimed to prepare a gelatin-based hydrogel and to characterize it for therapeutic purposes.

Methods: In this experimental-laboratory study, a gelatin hydrogel was made using a microbial transglutaminase (mTG) enzyme. The prepared hydrogel was evaluated in terms of appearance, physical, and chemical properties. To investigate the biological properties of the hydrogel, cells were cultured on it and the toxicity of the hydrogel for the cells was investigated. The location of the cells on the hydrogel was imaged via an electron microscope. The absorption and reflectance characteristics of the hydrogel were recorded by optical spectroscopy. Data were collected and statistical analysis was performed.

Results: The results showed that the mTG gelatin hydrogel had a uniform pore size and good physical, chemical, and mechanical properties for use in wound healing. Cell experiments showed evident cell proliferation and high viability. The results also revealed that the cells grew vigorously and adhered tightly to the hydrogel.

Conclusion: The preparation of a gelatin hydrogel under GMP conditions can be considered in the healing of diabetic wounds and burns.

Keywords: Dressing; Gelatin; Hydrogel; Tissue engineering; Wound.
\end{abstract}

\section{Introduction}

Wounds can be classified based on their cause of occurrence, depth, healing time, shape, and complexity. Wounds are generally categorized into acute and chronic. In the acute type of wound, it heals within 12 weeks, but in the chronic type, more time is needed., ${ }^{1,2}$ Wound healing stages are four successive stages that include homeostasis, inflammation, proliferation, and puberty. Although the wound healing process is linear, sometimes the healing process can be reversed, which depends on the patient's internal or external conditions. Failure in the wound healing process can lead to the development of a chronic wound. Factors that contribute to chronic wounds include venous disease, infection, diabetes, and metabolic disorders in adulthood. ${ }^{3}$ Careful wound care can speed up the wound healing process. It is possible to reduce the possibility of wound recurrence by maintaining moisture and cleanliness, taking care of the injury, and preventing

Please cite this article as follows: Nilforoushzadeh E, Behtash Amiri A, Shaghaghi B, Alimohammadi A, Ahmadi R, Khodaverdi Darian E, et al. Characterization of an enzyme-catalyzed crosslinkable hydrogel as a wound dressing in skin tissue engineering. J Lasers Med Sci. 2021;12:e77. doi:10.34172/jlms.2021.77. 
infection. Various dressings are used to heal acute wounds to protect against infection, whereby the wound heals naturally. In the case of chronic wounds, due to the loss of biological signals, specific treatment methods should be used to heal. ${ }^{4}$

One of the effective factors in wound healing is the use of an appropriate wound dressing. The wound dressing replaces the epithelium of the skin that has been damaged by the injury. The use of a wound dressing can greatly shorten the wound healing process. Despite many advances in medical engineering and biomaterials, wound dressings that can meet the broad needs of patients are not yet available to the healthcare team and patients. The ideal wound dressing can be used for different types of patients, regardless of the location and type of wound. This ideal wound dressing has properties such as keeping the wound environment moist, absorbing secretions, preventing the entry of microorganisms, and the ability to exchange gases. The materials used in this wound dressing should not be allergenic or toxic and should be adhesive to the cells (unless it is biodegradable) so that it can be removed from the wound without creating a new wound. This wound dressing should also improve the wound healing process and be cost-effective. ${ }^{5}$

The wound dressing can be divided into passive, active, advanced, and bioactive types. Passive wound dressings are made of simple fibers of fabric that are placed on the wound and have the minimum effect on the wound. Active wound dressings are made of hydrogel and sponge material and provide water plus oxygen for the wound. Advanced and bioactive wound dressings are composed of substances such as hydrocolloids, alginates, and collagen which can have a direct effect on wound healing. ${ }^{1,6,7}$

Regarding the potential applications of natural and synthetic hydrogels in the clinical field, and as gelatin is one of the most important natural polymers readily available, and since stem cells need a scaffold that would play a transferable role for them, and given the fact that there are several methods of evaluating the structure of the scaffold, the present study prepared and characterized gelatin-based hydrogels to enable the use of this product as a wound dressing.

\section{Materials and Methods \\ Preparation of Hydrogels}

Gelatin (Type A, Sigma, USA) was prepared at concentrations of $10 \%$ and $7 \%$ weight/volume $(\mathrm{w} / \mathrm{v})$ and transferred to bain-marie at $50^{\circ} \mathrm{C}$ for 30 minutes. The microbial transglutaminase (mTG) enzyme with a concentration of $10 \%$ was used as a cross-linker to make crosslinks (transverse connections) between gelatin strands, and it was transferred to the incubator for 24 hours to form crosslinks of gelatin chains. A hydrogel prepared by physical method or thermal-cooling method was used to control the sample. For this purpose, the prepared gelatin solution was transferred to the desired container and exposed to $4^{\circ} \mathrm{C}$. The prepared hydrogel was placed in a freeze dryer in two methods for freeze drying. ${ }^{8}$

\section{Fourier Transform Infrared Spectroscopy (FTIR)}

To identify the substance and to determine the different functional groups in the chemical structure of the hydrogel, FTIR method was used. To do this, the hydrogel was powdered using liquid nitrogen and was subjected to FTIR spectroscopy after mixing with potassium bromide powder. ${ }^{9}$

\section{Contact Angle Analysis}

The purpose of this work was to examine the surface properties of hydrogels in the vicinity of the biological environment and the rate of hydrophilicity of the samples, along with the reaction between it and the environment, cell adhesion, and so on. To this aim, based on the static sessile drop method, a drop of water was placed on the hydrogel and imaged with the device. The equilibrium contact angle created between the water droplet edge and the hydrogel surface was calculated by a goniometer. ${ }^{10}$

\section{Hydrogel Reflectance Spectroscopy}

The optical spectra of hydrogel absorption were recorded using an optical spectroscopy system equipped with optical fiber (Ocean optics, USB2000). For the spectral study, a halogen-tungsten light source was employed in the visible spectral region with a wavelength of 400-700 $\mathrm{nm}$. Source light was sent and collected by an optical fiber to the evaluated hydrogel samples. ${ }^{11,12}$

\section{Culture of Cells on Hydrogels}

The aim of this study was to investigate the potential of hydrogels in producing artificial skin and the ability to culture cells on it. For this purpose, the hydrogel was prepared in the desired plate and sterilized with UV light under the hood for 1 hour. Fibroblast cells available in the bank were used. After reaching the desired density, the cells were trypsinized and transferred to the hydrogel. The cell-containing hydrogel was placed in an incubator with the environment changed daily and evaluated under a microscope. ${ }^{9}$

\section{Investigation of Cytotoxicity of Hydrogels for Cells}

For evaluating the toxicity of hydrogels for cells, the MTT test was used. Hydrogels were prepared in 96-well plates and fibroblast cells were cultured at a density of $10^{5}$ in each well. After 48 hours, the culture medium was drained with $100 \mu \mathrm{L}$ of DMSO poured on each sump and placed in the incubator for 5 hours. Dimethyl sulfoxide (DMSO) was then drained and the 3-(4,5-dimethylthiazol-2-yl)2,5-diphenyl-2H-tetrazolium bromide (MTT) solution was poured onto the cells for 10 minutes and it was further read by the Elisa Reader at $545 \mathrm{~nm} .{ }^{13}$ 


\section{Imaging of Cells Cultured on Hydrogels by Electron} Microscopy

After fixation with glutaraldehyde and drying in room air, cutting and coating with gold, the hydrogel was imaged with scanning electron microscopy (SEM) imaging to investigate how the cultured cells were placed on the hydrogel. ${ }^{14}$

\section{Results}

\section{Hydrogel Morphology}

The results revealed that the low concentration of gelatin (4\%) did not have enough strength and consistency for transfer. Concentrations of 7 and greater than $10 \%$ gelatin had good consistency. The color of the samples was milky and had relative transparency (Figure 1).

\section{Analysis of FTIR Results}

The results of the FTIR test and comparison of the spectra obtained from gelatin powder and cross-linked gelatin with transglutaminase with different percentages showed the molecular changes of gelatin in its three-dimensional process with the enzyme. In all samples, absorption within the range of 1600 to $1700 \mathrm{CM}^{-1}$ indicated tensile absorption of the $\mathrm{C}=\mathrm{O}$ group in type 1 amide bond, 1500 to 1590 revealed the flexural absorption of the $\mathrm{NH}$ group attached to $\mathrm{CH}$ in type II amide bond, and $1200 \mathrm{CM}^{-1}$ showed tensile absorption of the $\mathrm{CN}$ group and flexion of the $\mathrm{NH}$ group in type III amide bond in the main gelatin chain.

Absorption in the region of about $3500 \mathrm{CM}^{-1}$ indicated the tensile peak of $\mathrm{OH}$ and $\mathrm{NH}$ amide groups. The location of these absorbents in the gelatin powder and hydrogel was constant and only the peak intensity increased, suggesting that the gelatin chains in the hydrogel were healthy. A slight change in the absorption of type II-amide-related groups was at around $1500 \mathrm{CM}^{-1}$, a change in the $\mathrm{NH}$ groups related to type III-amide, and a peak intensification related to absorption in the region of about 1070 and $1030 \mathrm{CM}^{-1}$ signaled the formation of crosslinks. Increasing the peak intensity of the enzyme by $10 \%$ compared to $7 \%$ suggested an increase in crosslinking in these hydrogels. It can be concluded that the crosslinking in the hydrogel increased with increasing

(A)

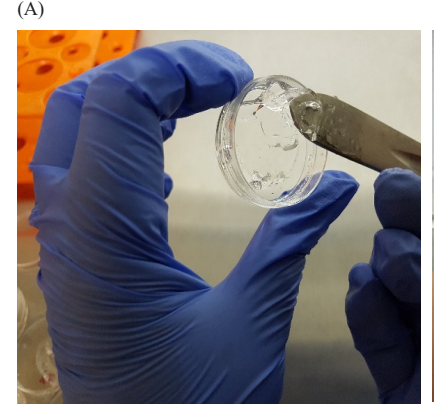

(B)

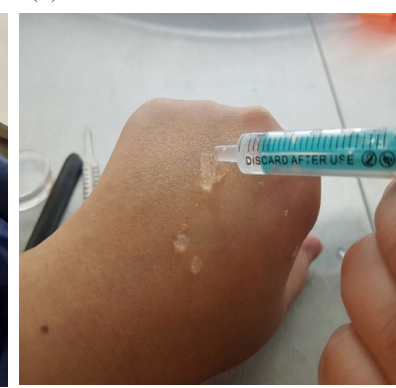

Figure 1. Morphology of the Gelatin Hydrogel Prepared With the Enzymatic Cross-Linker (mTG). the concentration of the cross-linker agent (Figure 2).

\section{Analysis of Contact Angle Results}

Based on the obtained results, it was found that in physical and enzymatic methods, the hydrogels made with a lower percentage of gelatin (7\%) had a lower contact angle and as a result more hydrophilic properties compared to hydrogels made with a higher percentage of gelatin (10\%). Higher hydrophilic properties can indicate that most of the functional groups involved in the hydrogen bond are free. In hydrogels made with a lower percentage of gelatin, due to the greater distance between the chains and the availability of more bonding groups, the hydrophilicity was higher. Whatever the size of surface cavities in the hydrogel is larger, a larger hydrophilic surface area is available, and more functional groups are available for water to form hydrogen bonds. In addition, in hydrogels made with a higher percentage of gelatin, more functional groups are involved in crosslinking; as a result, fewer functional groups are available for hydrogen bonding with water.

When comparing the hydrogels made by physical and enzymatic methods, it was observed that the difference in hydrophilicity between the two hydrogels with different percentages in the physical method (62.3 and 123.1) was far greater than that in the enzymatic method (82.7 and 88.8). In the case of hydrogels made with a concentration of $10 \%$ gelatin by the physical method, compared to hydrogels made by the same concentration by the enzymatic method, due to the nature of bonding, which is based on hydrogen bonds, the groups involved in hydrogen bonding bonded together and fewer groups were left to bond with the water making the surface of the hydrogel almost hydrophobic. In the hydrogel made with a concentration of $7 \%$ gelatin via the physical method, compared to hydrogels made with the same concentration by the enzymatic method, as observed in

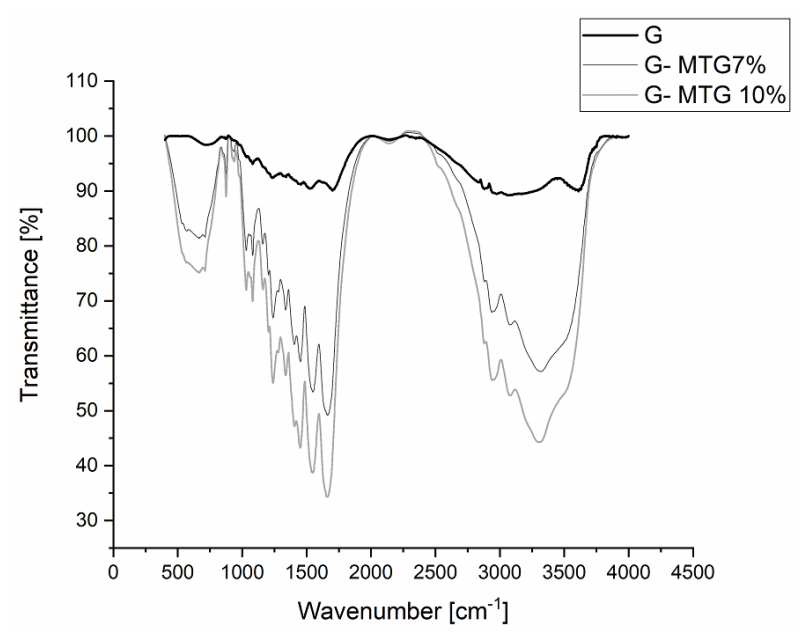

Figure 2. FTIR Spectrum of Gelatin Compared to Cross-Linked Gelatin With 7\% Transglutaminase Enzyme (7\% G-mTG) and 10\% Transglutaminase Enzyme (10\% G-mTG). 
the SEM observations, far fewer chains were involved in hydrogen bonding and more functional groups remained free to enter the hydrogen bond with the water, where the surface of the hydrogel was more hydrophilic.

Since the surfaces with a contact angle between zero and 90 degrees are considered as highly wettable and hydrophilic, hydrogels made by the enzymatic method and the physical method with a concentration of $7 \%$ are considered hydrophilic (Figure 3).

\section{Analysis of Spectroscopic Results}

Based on the optical absorption spectra in the near visible-infrared range, the synthesized hydrogels had an absorption peak with a wavelength of about $950 \mathrm{~nm}$. Depending on the amount of water these hydrogels contain, these absorption peaks were evaluated as stronger or weaker than each other. Meanwhile, it seemed that the absorption of these hydrogels was increasing toward the wavelengths higher than $1000 \mathrm{~nm}$, which corresponded to the water absorption peak in the infrared region

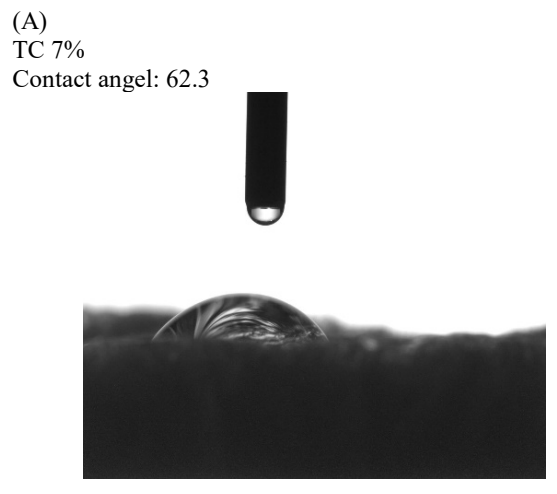

(C)

mTG 7\%

Contact angel: 82.7
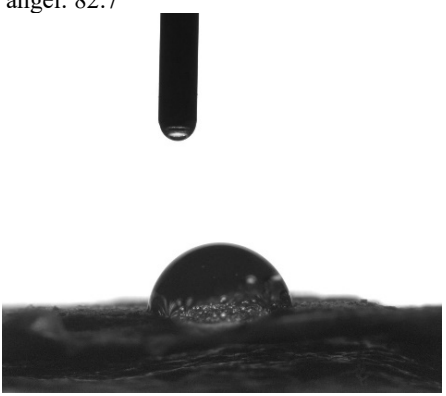

(E)

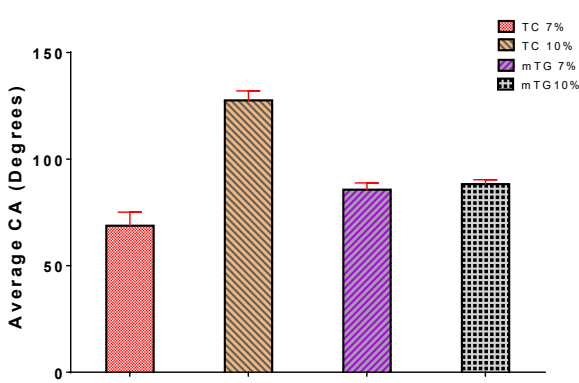

(Figure 4).

\section{Microscopic Examination, Cell Growth and Adhesion}

The results of cell culture on the hydrogel showed that the cells were round-shaped on the first and second days of transfer and spindle-shaped on the following days (Figure 5A).

\section{MTT Test Results}

The results of the MTT test indicated that the hydrogel prepared by the enzymatic method had no toxicity for the cells and the cells had proper growth as well as proliferation on this scaffold (Figure 5B).

\section{SEM Images of Cell Cultures on Hydrogels}

The results showed that the cells cultured on gelatin were visible and cultured uniformly (Figure 5C).

\section{Discussion}

Gelatin is a biopolymer, a product of partial hydrolysis of

(B)

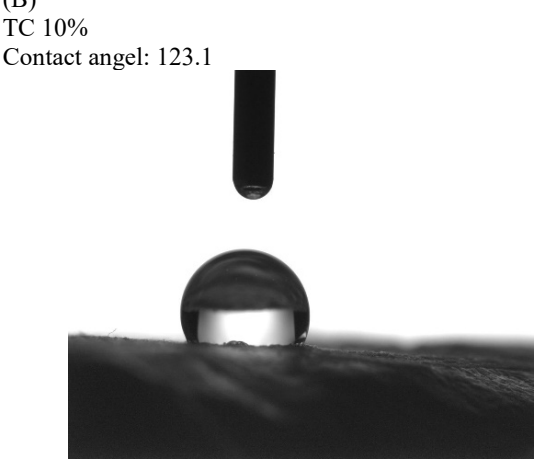

(D)

mTG $10 \%$

Contact angel: 76.5
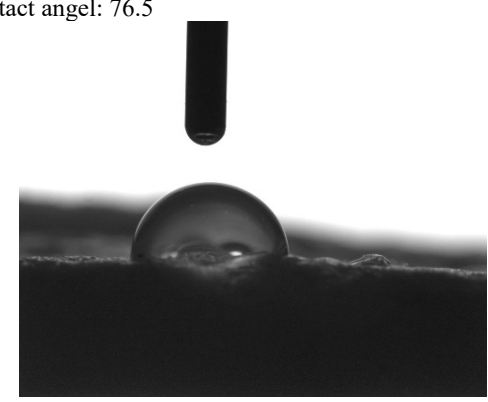

Figure 3. Results of Contact Angle Analysis of the Hydrogel Prepared by Two Physical (A, B) and Enzymatic Methods (C, D) With Concentrations of 7 and $10 \%$. Average contact angle analysis of the hydrogel prepared by two methods (E). Note: TC, thermal cooling. 
(A)

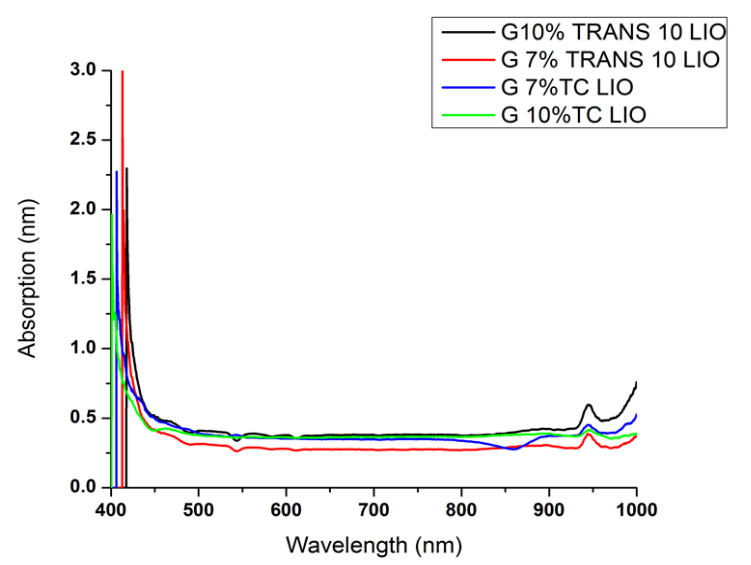

(B)

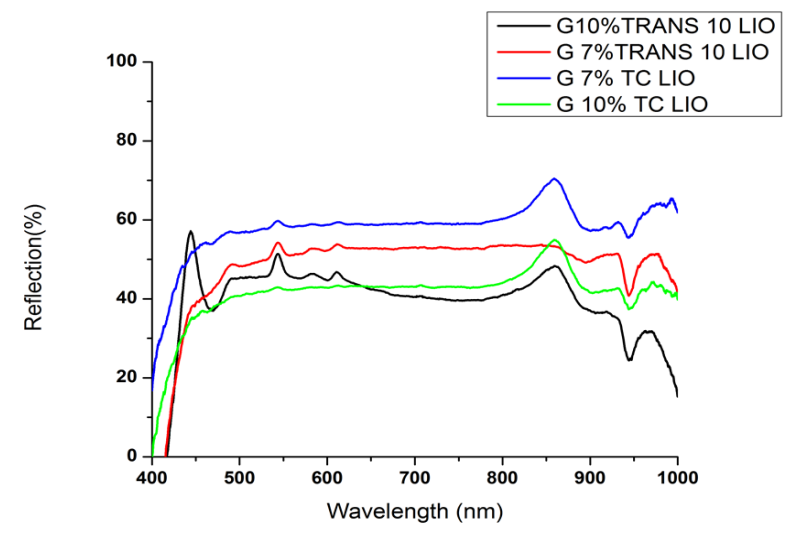

Figure 4. Absorption Spectrum (A) and Reflectance Spectrum (B) of the Hydrogel Prepared by the Thermal-Cooling Method With a Concentration of $7 \%$ (TC 7\%), the Hydrogel Prepared by the Thermal-Cold Method at 10\% (TC 10\%), the Hydrogel Prepared by the Enzymatic Method at 7\% (mTG 7\%), and the Hydrogel Prepared by the Enzymatic Method at 10\% (mTG 10\%) in the Range of Visible Light Wavelengths of 400-1000 nm. Note: TC, thermal cooling.

collagen, which is widely used in biological, medical, and pharmaceutical applications due to its biocompatibility, biodegradability, non-toxicity, and non-carcinogenicity in its structure. Gelatin is used extensively in medications, reconstructive medicine, and tissue engineering. If a three-dimensional substrate and a cellular scaffold structure are required, gelatin can be converted into a three-dimensional porous sponge via various methods. The porous gelatin sponge provides a proper substrate for cells to settle and grow so that they can adhere to it and grow. Permanent covalent or temporary hydrogen bonds can be used to make this three-dimensional substrate. Using chemical molecules or biological enzymes, controlled covalent cross-links are created between the polymer chains to stabilize the so-called three-dimensional polymer. Liquid gelatin can also be converted to gel using hydrogen bonds created between adjacent chains in the heat-cold method.

The transglutaminase enzyme is widely used for its

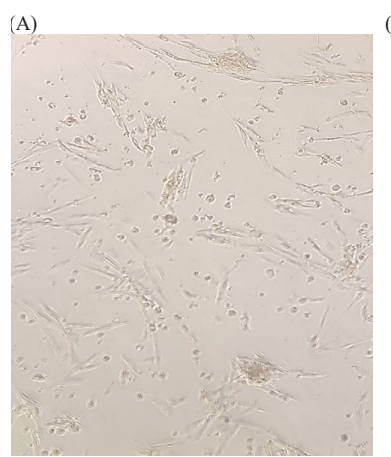

(B)

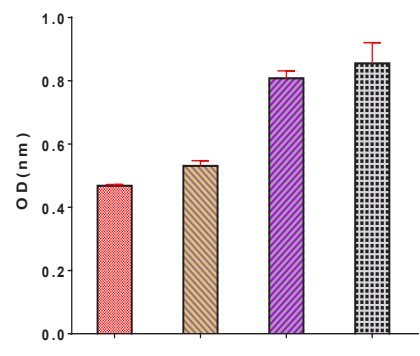

(C)

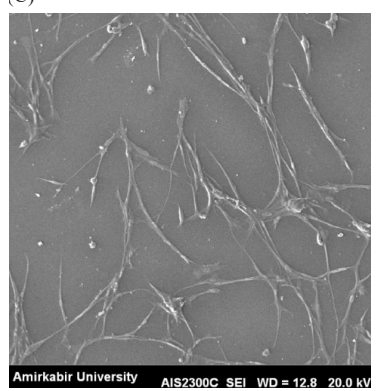

Figure 5: Spindle Morphology of Cells on 10\% Hydrogel Prepared by the Enzymatic method ( $\times 10$ magnification) $(A), O D$ of Different Concentrations of Hydrogels (B), SEM Images of Cells at $10 \%$ Concentration on Day $4(300 \mu \mathrm{m})(\mathrm{C})$. Note: OD, optical density.

ability to make cross-linking in proteins. This enzyme catalyzes the acyl transfer reaction between the glutamine $\lambda$-carboxyamide group (acyl donor) and the $\varepsilon$-amine lysine group (acyl receptor) to form $\varepsilon$ - ( $\lambda$-glutaminyl) lysine between the two chains to create crosslinking between the protein chains. The $3 \mathrm{D}$ gelatin made using this enzyme has good biocompatibility. ${ }^{15}$

In the present study, hydrogels prepared with microbial transglutaminase had the appropriate properties of a biological dressing. Examination of the gelatin hydrogel under a microscope showed that the cells cultured on it had a routine morphology and natural life and these cells were adhesively and evenly distributed on the surface of the membrane. This suggests that gelatin hydrogel is a suitable substrate for culturing and preserving cells in the wound area.

One of the indicators for determining the nature of hydrogels is based on the chemical properties obtained based on FTIR data. In this study, increasing the peak intensity of the enzyme by $10 \%$ compared to $7 \%$ showed an increase in the cross-linking rate in these hydrogels. It can be concluded that with increasing the concentration of the cross-linking agent, the cross-linking in the hydrogel increased. Based on the results obtained from the contact angle study, it was found that hydrogels made with a concentration of $7 \%$ are considered hydrophilic compared to a higher concentration. Also, fibroblast cells were cultured successfully on the gelatin hydrogel and no toxicity was observed. SEM images showed the appropriate and expected morphology of the cells. 
Previous studies have confirmed the results of this study. 8,15

Another indicator for determining the nature of hydrogels is based on optical absorption spectra in the near visible-infrared range. The results of spectroscopy showed that the synthesized hydrogels had an absorption peak with a wavelength of about $950 \mathrm{~nm}$. Also, based on the amount of water these hydrogels contain, these absorption peaks were evaluated as stronger or weaker than each other. Meanwhile, the absorption of these hydrogels seemed to be increasing toward wavelengths higher than $1000 \mathrm{~nm}$, which corresponded to the water absorption peak in the infrared region.

The present study was conducted to investigate the chemical, physical, reflectance spectroscopy and biocompatibility properties of this hydrogel scaffold. This research is associated with limitations in terms of in vivo studies and animal models, and it is hoped that in the near future, it will be possible to study on a larger scale to decide on the use of this simple and cost-effective hydrogel as a wound healing dressing.

\section{Conclusion}

Overall, the results of this study revealed that the method used in this study is a reliable method for preparing gelatin hydrogel and has the potential to be used as a biological dressing in the treatment of wounds.

\section{Ethical Considerations}

The study protocol was approved by the Medical Ethics Committee of Tehran University of Medical Sciences (IR. TUMS.VCR.REC.1397.506) and also by the Forensic Medicine Organization of the country (IR.LMO.REC.1397.021).

\section{Conflict of Interests}

The authors declare that they have no conflict of interest.

\begin{abstract}
Acknowledgments
This work was supported by the 1) Skin and Stem Cell Research Center, Tehran University of Medical Sciences, 2) National Animal Modeling Network and in vivo Research, Council for Development of Stem Cell Sciences and Technologies, Vice -Presidency for Science and Technology, grant number 98/11626 and 3) Bio-photonic Group, Photonics of Organic Materials and Polymers (POMP) Laboratory, Laser and Plasma Research Institute, Shahid Beheshti University. The spiritual and financial support of these centers is hereby appreciated.
\end{abstract}

\section{References}

1. Zahedi P, Rezaeian I, Ranaei-Siadat SO, Jafari SH, Supaphol P. A review on wound dressings with an emphasis on electrospun nanofibrous polymeric bandages. Polym Adv Techno. 2010;21(2):77-95. https://doi.org/10.1002/ pat.1625.

2. Ferreira MC, Tuma P, Jr., Carvalho VF, Kamamoto F. Complex wounds. Clinics (Sao Paulo). 2006;61(6):571-8. https://doi.org/:10.1590/s1807-59322006000600014
3. Moore K, McCallion R, Searle RJ, Stacey MC, Harding KG. Prediction and monitoring the therapeutic response of chronic dermal wounds. Int Wound J. 2006;3(2):89-96. https://doi.org/:10.1111/j.1742-4801.2006.00212.x.

4. Thomas J. Wound healing and ulcers of the skin. Diagnosis and therapy-the practical approach. By A Shai and HI Maibach. (c) Springer-Verlag Berlin Heidelberg, 2005. ISBN: 35402127520470 848987. Hardcover, 270 pages. Pract Diabetes Int. 2005;22(8):283. https://doi.org/10.1002/ pdi. 848

5. Jayakumar R, Prabaharan M, Sudheesh Kumar PT, Nair SV, Tamura H. Biomaterials based on chitin and chitosan in wound dressing applications. Biotechnol Adv. 2011;29(3):322-37. https://doi.org/:10.1016/j. biotechadv.2011.01.005.

6. Abrigo M, McArthur SL, Kingshott P. Electrospun nanofibers as dressings for chronic wound care: advances, challenges, and future prospects. Macromol Biosci. 2014;14(6):772-92. https://doi.org/:10.1002/ mabi.201300561.

7. Daunton C, Kothari S, Smith L, Steele D. A history of materials and practices for wound management. Wound Pract Res. 2012;20(4)174-186.

8. Yang G, Xiao Z, Long H, Ma K, Zhang J, Ren X, et al. Assessment of the characteristics and biocompatibility of gelatin sponge scaffolds prepared by various crosslinking methods. Sci Rep. 2018;8(1):1-3. doi:10.1038/s41598-01820006-y

9. Perkasa DP, Erizal E, Purwanti T, Tontowi AE. Characterization of Semi-Interpenetrated Network Alginate/Gelatin Wound Dressing Crosslinked at Sol Phase. Indones J Chem. 2018;18(2):367-75. doi:10.22146/ IJC. 25710

10. Serrano-Aroca Á. Enhancement of hydrogels' properties for biomedical applications: Latest achievements. Hydrogels: IntechOpen; 2018. p. 91. doi:10.5772/intechopen.71671

11. Babadi M, Mohajerani E, Ataie-Fashtami L, Zand N, Shirkavand A. Quantitative Analysis of Skin Erythema Due to Laser Hair Removal: A Diffusion Optical Spectroscopy Analysis. JLasers Med Sci.2019;10(2):97-103. doi:10.15171/ jlms.2019.16.

12. Shirkavand A, Farivar S, Mohajerani E, Ataie-Fashtami L, Ghazimoradi MH. Non-invasive Reflectance Spectroscopy for Normal and Cancerous Skin Cells Refractive Index Determination: An In Vitro Study. Lasers Surg Med. 2019;51(8):742-50. https://doi.org/:10.1002/lsm.23095.

13. Yang G, Xiao Z, Long H, Ma K, Zhang J, Ren X, et al. Assessment of the characteristics and biocompatibility of gelatin sponge scaffolds prepared by various crosslinking methods. Sci Rep. 2018;8(1):1616. doi:10.1038/s41598-01820006-y.

14. Sisakht MM, Nilforoushzadeh MA, Verdi J, Banafshe HR, Naraghi ZS, Mortazavi-Tabatabaei SA. Fibrin-collagen hydrogel as a scaffold for dermoepidermal skin substitute, preparation and characterization. J Contemp Med Sci. 2019;5(1): 8-13. Doi:10.22317/jcms.v5i1.519.

15. Jaipan P, Nguyen A, Narayan RJ. Gelatin-based hydrogels for biomedical applications. Mrs Commun. 2017;7:416-26. doi:10.1557/mrc.2017.92. 